\title{
Advanced Suction Device with Continuous Oxygen Supply for Performing Meconium Suction and Identical Procedures
}

\author{
Kumar Ritesh', Bhavsar Raunak', C. Subramanian² \\ ${ }^{1}$ Department of Biomedical Engineering, VIT University, Vellore, India \\ ${ }^{2}$ Department of Mechanical Engineering, VIT University, Vellore, India \\ Email: ritesh.kumar2011@vit.ac.in, raunakbhavsar@gmail.com, subbu10123@gmail.com
}

Received 15 August 2014; revised 30 September 2014; accepted 15 October 2014

Copyright (C) 2014 by authors and Scientific Research Publishing Inc.

This work is licensed under the Creative Commons Attribution International License (CC BY). http://creativecommons.org/licenses/by/4.0/

\section{(c) (i) Open Access}

\begin{abstract}
Laryngoscopy is a medical procedure that provides a secure airway by passing a breathing tube through the mouth and into the lungs of a patient, and to perform meconium suction in newborns to avoid asphyxia. The ability to successfully perform meconium suction is highly dependent on operator skill and the availability of trained assistants to assist during the procedure. The immediate objective of this research paper is to present an improved device, so that the procedure can be performed at a faster pace and the need for assistants is eliminated. Experienced physicians have used the device presented in the paper on mannequin, and the operating time and ease were observed to have improved.
\end{abstract}

\section{Keywords}

Laryngoscopy, Meconium Aspiration Syndrome

\section{Introduction}

Meconium aspiration syndrome (MAS, alternatively "Neonatal aspiration of meconium") is a medical condition affecting newborn infants. It occurs when meconium is present in their lungs during or before delivery. Meconium is normally stored in the infant's intestines until after birth, but sometimes (often in response to fetal distress) it is expelled into the amniotic fluid prior to birth, or during labor. If the baby then inhales the contaminated fluid, respiratory problems may occur. In a study conducted between 1995 and 2002, MAS occurred in 1061 of 2,490,862 live births, reflecting an incidence of 0.43 of 1000 [1]. MAS requiring intubation occurs at higher rates in pregnancies beyond 40 weeks. $34 \%$ of all MAS cases born after 40 weeks required intubation compared to $16 \%$

How to cite this paper: Ritesh, K., Raunak, B. and Subramanian, C. (2014) Advanced Suction Device with Continuous Oxygen Supply for Performing Meconium Suction and Identical Procedures. J. Biomedical Science and Engineering, 7, 988-993. http://dx.doi.org/10.4236/jbise.2014.712096 
prior to 40 weeks. The passage of meconium-stained amniotic fluid (MSAF) during labor is reported to occur in $12 \%-15 \%$ of all live births. This figure is higher in deliveries associated with maternal complications (up to 24\%). Meconium aspiration syndrome (MAS) occurs in around 5\% of these pregnancies [2].

The meconium suction procedure needs to be performed under a 3-minute time frame to avoid severe brain damage. Since assistants are often not available; a doctor can rather perform the procedure independently if provided with the appropriate device. Assistance is primarily required during the procedure to hold the oxygen supply channel near the newborn and to control suction during the procedure. An instrumented laryngoscope made with medical grade stainless steel has been developed; it uses a mounted pathway for continuous oxygen administration from 0.5 to $3 \mathrm{~L} / \mathrm{min}$ and a controllable suction tubing which delivers -80 to $-120 \mathrm{~mm}$ Hg pressure is mounted on the laryngoscope to improve the operator's ease. This results in bringing the operating time well under 1 minute. Silicon tubing has been added to suction tube to prevent damage to the larynx or surrounding tissue during the interaction between the laryngoscope and the meconium surrounding the larynx of the patient. Based on scientific evidence, this project has the potential to save hundreds of thousands of lives through a simple and achievable mechanical innovation. The lack of trained professionals has to be overcome to save the millions of lives that are affected by MAS. The simplicity and ease of operation of the device is its USP.

\section{Background of Technology}

The current methods applied require two trained personnel who are often not available. Assistance and speed is crucial while performing the procedure since the procedure is time bound ( 3 min limit). An assistant holds an oxygen supply tube in front of the infant all the while, whereas another assistant handles the suction tube. Thus it takes three personnel to do the procedure. The equipment required for the management of meconium aspiration now includes the following:

- Laryngoscope with blade (size 1 for a term infant)

- Uncuffed endotracheal tube (size 3.5 - 4.0 for a term infant)

- DeLee suction catheter (12 - 14 French)

- Meconium aspirator

- Suction tubing

- Medical suction device set to a continuous pressure of -80 to $-120 \mathrm{~mm} \mathrm{Hg}$

For intubation and tracheal suctioning, the infant should be placed on a flat surface, ideally under a radiant warmer. The head should be in midline position, with the neck slightly extended into the so-called sniffing position. Placing a roll of towels under the infant's shoulders may help maintain slight neck extension. Do not flex or hyperextend the neck; this raises the glottis above the line of sight and makes intubation more difficult. Hold the laryngoscope in the left hand, and stabilizes the infant's head with the right hand. Introduce the laryngoscope blade into the right side of the mouth, and sweep the tongue to the left with the laryngoscope blade. To make introduction of the laryngoscope blade easier, try using the right index finger to open the newborn's mouth. Advance the laryngoscope blade until the tip is positioned on top of the epiglottis or immediately anterior to the epiglottis in the vallecula. Gently lift the laryngoscope blade upward to elevate the epiglottis and tongue to reveal the vocal cords. Do not "rock back" with the laryngoscope blade; doing so may damage the alveolar ridge. Identify the vocal cords, and attempt to verify the presence of meconium below the level of the cords. If meconium is present, the posterior pharynx may have to be suctioned with a DeLee suction catheter to improve visualization of the cords. Maintain direct visualization of the vocal cords as an assistant places the endotracheal tube into your right hand. Advance the endotracheal tube through open vocal cords until the vocal cord guide on the endotracheal tube is at the level of the cords. If the vocal cords are approximated, wait for them to open. Do not force the endotracheal tube through closed vocal cords; doing so can damage the cords. Stabilize the endotracheal tube in position by using an index finger to hold the tube against the hard palate. While holding the endotracheal tube in place, remove the laryngoscope blade. Ideally, the entire process of tracheal intubation should take less than 20 seconds. Attach a meconium aspirator, connected to a medical suction device supplying a continuous pressure of -80 to $-120 \mathrm{~mm} \mathrm{Hg}$, to the endotracheal tube. Occlude the suction-control port on the meconium aspirator to apply suction, applying suction, withdraw the endotracheal tube over a period of 3 - 5 seconds. If a substantial amount of meconium is returned by suction, intubation and suction should be repeated until the aspirated material is cleared or the newborn's heart rate falls below 100 beats/min [3]. 


\section{Solution}

A simple hand operated device that serves the function of the laryngoscope, Dee Lee catheter and the oxygen supply tube, all operable by a single professional is a massive aid to the professional. The design eliminates the need to have trained assistants which are often not available. The doctor has all the three functions available in the handheld device and doesn't lose valuable time in demanding and introducing the catheter after spotting the meconium at the back of the throat and making sure that a continuous oxygen supply is being provided to the patient in order to prevent hypoxia.

The international guidelines for meconium aspiration syndrome treatment and management recommend suctioning for infants with depressed respiratory effort, poor muscle tone, and/or heart rate $<100 \mathrm{beats} / \mathrm{min}$. The design of the device has been developed under the guidance of senior anesthetists. The design consists of a laryngoscope blade mounted with two passages. The first passage serves as a continuous supply outlet for oxygen. The second passage consists of a hand controlled suction tube which can be extended and retracted by the doctor via sliding it on the provided hinge. The suction is activated upon the closure of the pore provided at the doctor's end of the device (explained in the design section). Thus, the need of an assistant to hold the oxygen supply during the procedure is avoided. Also, the suction tube doesn't get stuck to the cheek midway the suction can be activated and deactivated by simply closing and opening the pore respectively. The personnel performing the procedure are always in control of the whole procedure with no dependence on an assistant and other complication due to multiple parameters that cost time

\section{Methods}

\subsection{Construction and Design Parameters}

The entire laryngoscope and all its accessories are made up of stainless steel. It is used to avoid corrosion over long term use. Small supports (Parts labeled 6 and 9; Figure 1) were welded to the side of the blade so that the suction tube (labeled 7; Figure 1) can be moved with ease while the performing the operation. The dimensions of the blade have been chosen as per a standard infant-miller blade [4]. The length of the suction tube is about $6.5 \mathrm{~cm}$ it is as per the average depth up to which the tube has to be inserted. The diameter of the suction tube is 3 $\mathrm{mm}$ this parameter has been chosen after careful consideration of the viscosity of the fluid to be removed, amount of suction produced, diameter of the orifice (labeled 5; Figure 1). The diameter of the orifice used to control the suction is about $3 \mathrm{~mm}$. The plate in which the suction orifice is present is designed such that it is slightly bent to better accommodate the pediatrician's thumb and improve comfort while performing the procedure. The plate thickness is $1 \mathrm{~mm}$ which is appropriate for welding it to the tube. The connectors are dimensioned according to the specification of the suction pump.

Care has been taken so that no leaks are present at any of the junctions. Leakage of air leads to loss in efficiency of the device at worst case may even lead to failure of the procedure. Finally the oxygen supply (labeled 2; Figure 1) is placed on the other side of the blade along with the LED (labeled 3; Figure 1) for better space management. It also helps the user better control the suction tube. The dimensions of the continuous oxygen supply are based considering parameters such as oxygen flow rate by the pump and amount required by the infant after the procedure. The oxygen supply tube is $2 \mathrm{~mm}$ in diameter. The handle of the laryngoscope is knurled for a better grip and is also designed to contain the battery which powers the LED. As the blade is of standard dimension it is detachable from the handle and is also easily replaced with another kind of blade for a different procedure [4].

\subsection{Description of the Design}

A complete understanding of the invention will be obtained from the following disclosure taken in conjunction with the accompanying drawings in which:

Figure 1 is a perspective view of a preferred embodiment of the invention; the part labeled $\mathbf{1}$ is the retracting blade which is used to retract the larynx while performing the procedure and the part labeled $\mathbf{3}$ is a LED i.e. light emitting diode which provides illumination to view and detect the presence of meconium. The part labeled $\mathbf{2}$ is the continuous oxygen supply pathway which is connected to the oxygen supply along the base connector labeled 11. Hence; the patient takes in oxygen in the first gasp. The part labeled $\mathbf{4}$ is the suction tube plate that is used to hold the suction tube and apply force to move the suction into and away from the mouth while operat- 
ing. The labeled 5 is the suction valve used to control the flow of air out. The labeled 7 is the suction tube and attached from its front end is the labeled $\mathbf{1 0}$ silicon tube. If left open the suction valve sucks in the air from the surrounding through the pore and prevents suction through the tube from the front end terminal. In order to activate suction at the terminal labeled 12 the valve has to be closed with the help of the thumb. Rear support labeled 6 holds the mounted suction tube in place and allows the movement of the suction tube in forward and backward direction without losing hold of the suction tube. It also prevents the removal of the suction tube from the retracting blade when the rubber stopper labeled $\mathbf{8}$ hits it. The rubber lock labeled $\mathbf{8}$ is responsible for limiting the backward motion of the suction tube upon hitting the rear support labeled $\mathbf{6}$ so that it does not get detached from the retracting blade. The rubber lock is not permanently fixed to the suction tube it can be moved and adjusted but the rubber lock is tight enough so that it cannot be moved until a large force is applied. The part labeled 9 is the forward support for the silicone tube labeled 10 to be mounted on the suction tube. Suction tube labeled 7 is the pathway via which the meconium is driven out of the patient's body. The suction tube needs to be activated by closing the suction valve labeled $\mathbf{5}$ with the thumb. A disposable silicon tube labeled $\mathbf{1 0}$ is attached at the head of the suction tube to avoid damage to the larynx by the rigid suction tube. This can be replaced after every use. Figure 2 is a back view of an embodiment of the device. Figure 3 is a left side view of the device. Figure 4 \& Figure 5 is right side and the top view of the design respectively. The novelty resides in the shape and configuration of the article as illustrated.

\section{Results and Discussions}

The silicon sleeve at the terminal of the suction tube provides a smooth and soft surface that comes in contact with the infant and thus minimizes the risk of damage. Unlike the present technology that provides a continuous suction pressure, the new device provides a continuous suction pressure of -80 to $-120 \mathrm{~mm} \mathrm{Hg}$ at the terminal which can be deactivated and activated simply by placing and removing the thumb over the suction valve. Instead of investing time in placing the oxygen tube and having an assistant monitor the placement constantly, the

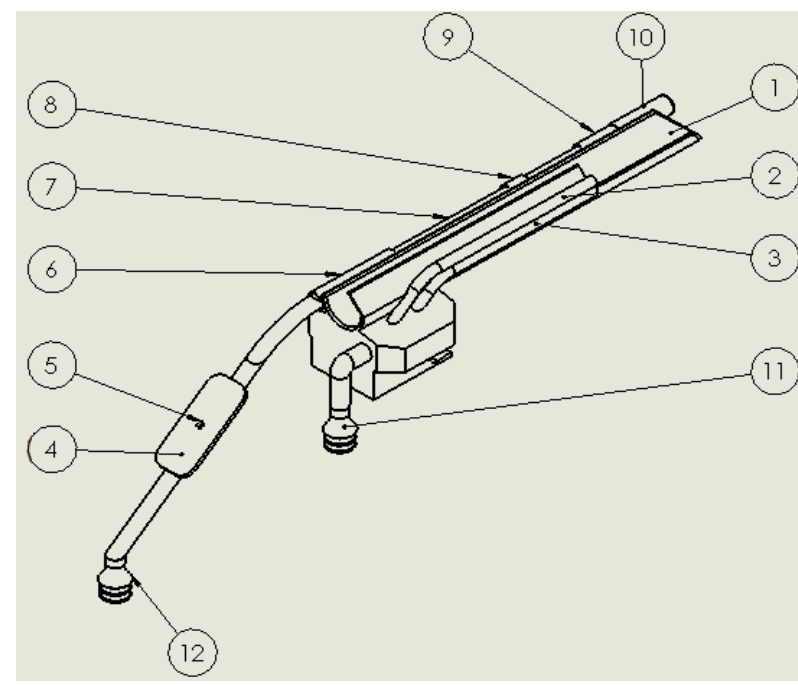

Figure 1. Perspective view.

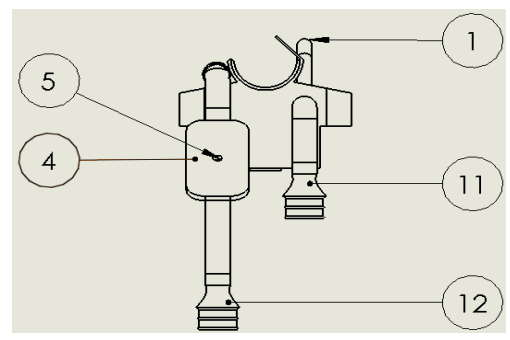

Figure 2. Back view. 


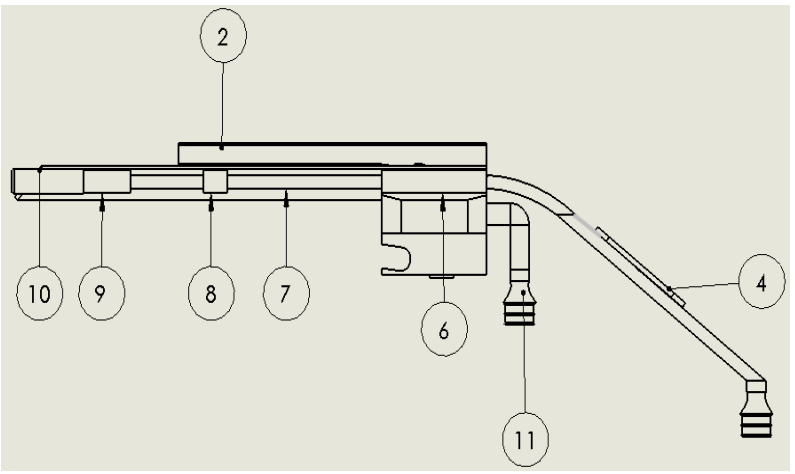

Figure 3. Left side view.

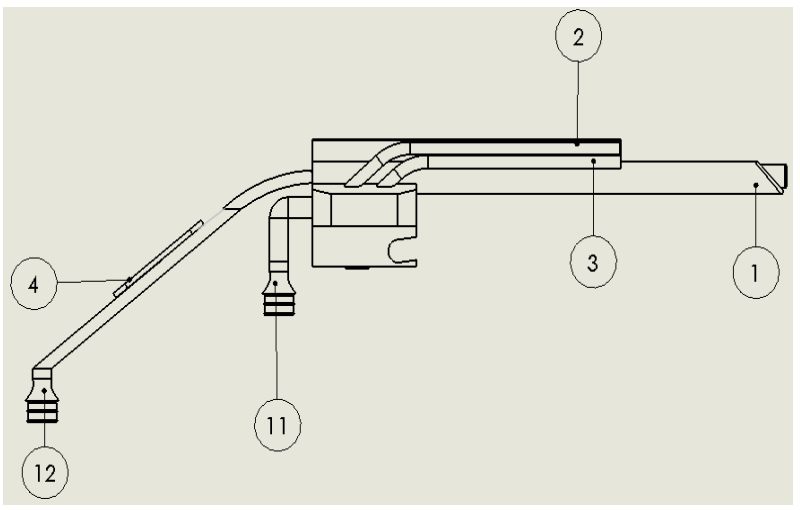

Figure 4. Right side view.

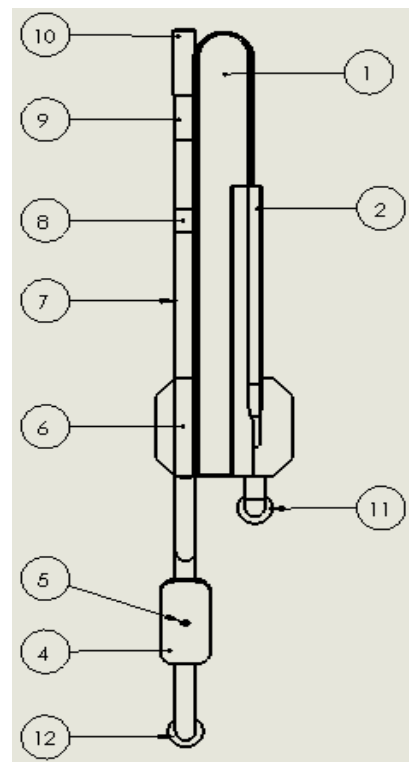

Figure 5. Top view.

continuous oxygen supply tube of the new device can directly administer up to $3 \mathrm{~L} / \mathrm{min}$ output to keep oxygen available to the infant at all times during the procedure and facilitate maximum delivery of oxygen to the hypoxic infant without requiring an assistant to monitor it. A white LED provides better illumination for the oral cavity to provide better visibility to the doctor unlike the yellow LED which has lower illumination capacity.

It is clear that the suction tube can be used to clear the meconium without causing any damage and without the 
need of an assistant to regulate the suction. Controlled suction allows unnecessary withdrawal of the accumulated oxygen in the oral cavity of the infant. This ultimately results in bringing down the operating time to under a minute and makes the doctor independent of the assistants.

\section{Conclusion}

With the proposed design of the meconium suction device, an integrated system for controlled suction, continuous oxygen supply and laryngoscopy was achieved. The suction valve facilitates the use of suction at the doctor's discretion and avoids unnecessary vacuum. The continuous oxygen supply keeps oxygen available to the infant at all times. The new device can be used for performing meconium suction procedure under the control of a single professional without the need of assistants. In this way, the proposed device can reduce the time required for the procedure, reduce errors due to untrained assistance and make the procedure economical.

\section{References}

[1] Usta, I., Mercer, B. and Sibai, B. (1995) Risk Factors for Meconium Aspiration Syndrome. Obstetrics and Gynecology, 86, 230-234. http://dx.doi.org/10.1016/0029-7844(95)00124-A

[2] Anwar, Z., Butt, T.K., Anjum, F. and Kazi, M.Y. (2011) Mortality in Meconium Aspiration Syndrome in Hospitalized Babies. Journal of the College of Physicians and Surgeons Pakistan, 21, 695-699.

[3] Sawyer, T.L., Rosenkrantz, T., et al. (2013) Intubation and Tracheal Suctioning for Meconium Aspiration.

[4] Miller, R.A. (1941) A New Laryngoscope. Anaesthesiology, 2, 318-320. http://dx.doi.org/10.1097/00000542-194105000-00008 
Scientific Research Publishing (SCIRP) is one of the largest Open Access journal publishers. It is currently publishing more than 200 open access, online, peer-reviewed journals covering a wide range of academic disciplines. SCIRP serves the worldwide academic communities and contributes to the progress and application of science with its publication.

Other selected journals from SCIRP are listed as below. Submit your manuscript to us via either submit@scirp.org or Online Submission Portal.
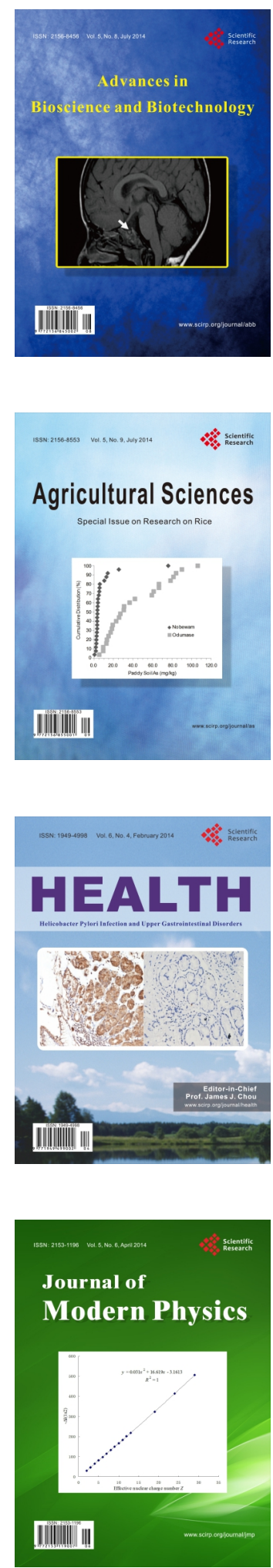
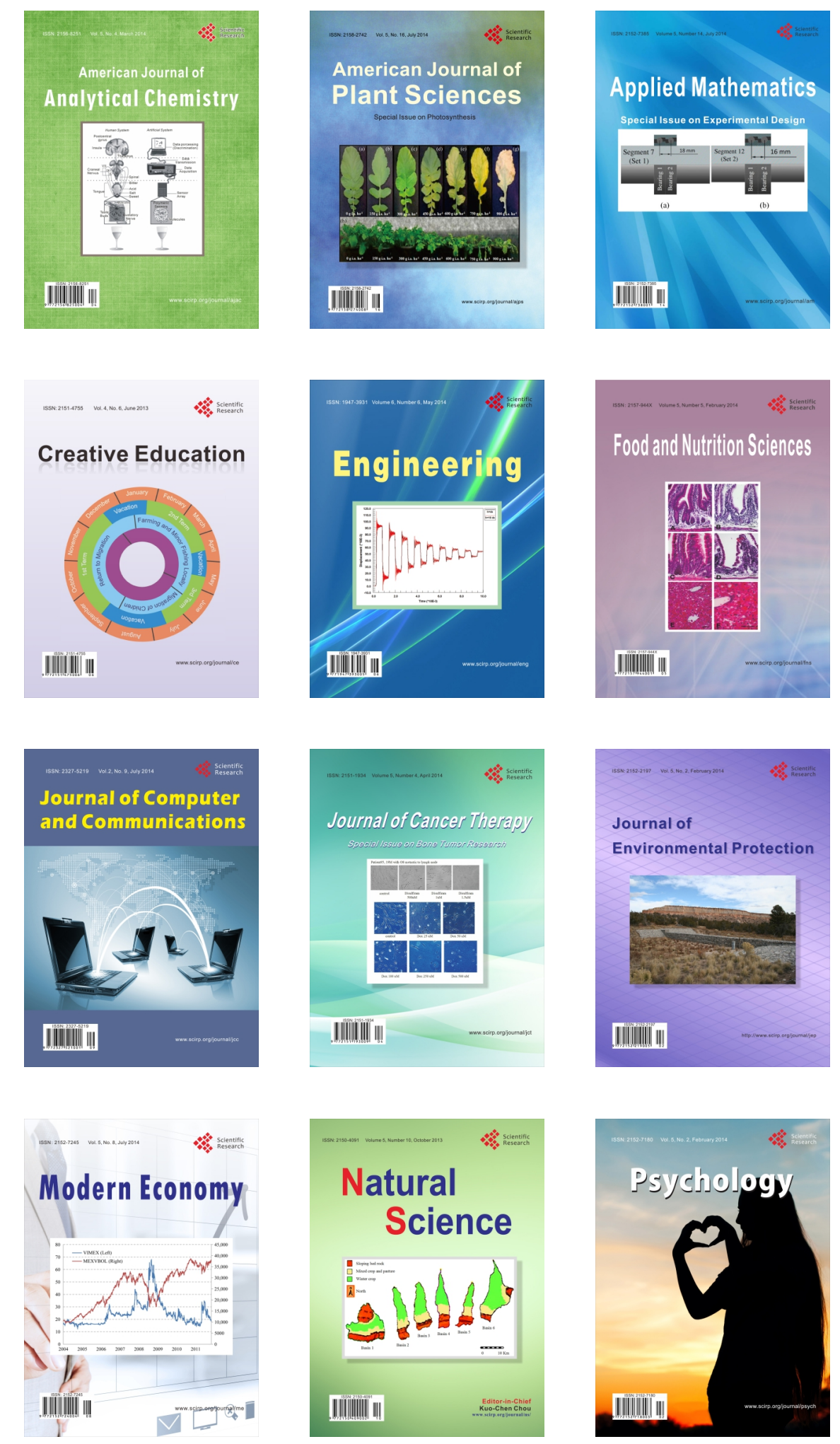